\title{
Experimental Detection of Transformer Excitation Asymmetry through the Analysis of the Magnetizing Current Harmonic Content
}

\author{
Nebojsa Pjevalica ${ }^{1}$, Nenad Petrovic ${ }^{2}$, Velibor Pjevalica ${ }^{3}$, Nikola Teslic $^{1}$ \\ ${ }^{I}$ Computing and Control Engineering Dept. University of Novi Sad, Faculty of Technical Sciences, \\ Trg Dositeja Obradovica 6, 21000 Novi Sad, Serbia \\ ${ }^{2}$ School of Electrical Engineering, \\ Stari grad, Visokog Stevana 37, 11000 Belgrade, Serbia \\ ${ }^{3}$ Technical Provision Section, \\ JP Srbijagas, Narodnog fronta St. 12, 21000 Novi Sad, Serbia \\ pjeva@uns.ac.rs
}

\begin{abstract}
The presence of a low DC voltage in a power grid produces an excitation asymmetry for the distribution transformers, resulting in unwanted half-cycle saturation that may impair transformer safety, reliability and efficiency, especially in increasing the reactive power, stray losses and iron losses. This paper highlights a diagnostic method based on the phase value of the particular even harmonics of a transformer excitation current as the basic marker of an excitation asymmetry. In order to achieve a straightforward insight into the nature of even harmonics behaviour, a novel method of decomposition an excitation current waveform into its unhysteretic (pure magnetization) and hysteretic components has been included in the harmonic analysis.
\end{abstract}

Index Terms-Decomposition; DC bias; harmonic analysis; hysteresis; magnetization; phase measurement; power transformer; transformer core.

\section{INTRODUCTION}

It is well known that the waveform of the transformer or reactor AC excitation current gives essential information concerning magnetic flux density B, magnetic strength field $\mathrm{H}$ or power losses of the related magnetic core. For the sinusoidal magnetic flux case, as it has been reported in [1], the waveform of the transformer or Epstein frame $\mathrm{AC}$ excitation current describes the related magnetic core behavior exactly in the same way as the corresponding hysteresis loop curve does. But, the present interest of the great importance to the researchers is to discover what reliable information, in diagnostic sense, could be extracted from the $\mathrm{AC}$ excitation current waveform in the presence of DC current bias.

Several well-known phenomena can cause the arising of DC current bias. The most common ones are the widespread use of the grid-connected static power converters [2], [3], the influence of the high-voltage DC transmission [4] and the geomagnetically induced current (GIC) [5], [6]. In such circumstances, when the mutual influence between DC and

Manuscript received 17 December, 2015; accepted 24 March, 2016.

This work was partially supported by the Ministry of Education, Science and Technological Development of the Republic of Serbia, under grant number: "TR32014".
AC flux exists, there is a coexistence of odd and even harmonics in AC excitation current. This coexistence is present in the transformer inrush current as well, as it has been reported in [7].

Among even harmonics of an $\mathrm{AC}$ excitation current in a steady state with DC bias, the second order harmonic exhibits some unique properties - an almost constant phase shift relative to the fundamental component and the phase jump of $\pi$ as the DC bias changes the sign [8]-[10].

Furthermore, the harmonic analysis of $\mathrm{AC}$ excitation current waveform, based on a very accurate sampling system, indices similar behaviour for the forth, sixth and eighth harmonics as well [11].

This paper aims to examine experimentally the robustness of the above-mentioned property in the real environment with a considerable presence of the noise. In addition, the paper presents a new approach to decomposition the AC excitation current waveform into its hysteretic and unhysteretic (pure magnetization) components.

\section{ASYMMETRIC EXCITATION AND HYSTERESIS B-H LOOP}

The non-sinusoidal waveform of the transformer $\mathrm{AC}$ excitation current in a steady state is the consequence of the nonlinear magnetic characteristics of the transformer core and the core losses. With the supplied voltage free of any DC component, even though the voltage waveform is not perfectly sinusoidal, a hysteresis loop of the transformer core is symmetrical to the $\mathrm{B}-\mathrm{H}$ coordinate system origin This fact implies the existence of the odd harmonics of an AC excitation current waveform exclusively.

The appearance of a DC component of the external magnetic field $\mathrm{H}$ will result in a certain distortion and shift of the existing B-H hysteresis loop toward the first or the third quadrants of the B-H coordinate system, in dependence of the positive or negative DC bias sign, as it is represented in Fig. 1. This will cause an arising of the even harmonics in the $\mathrm{AC}$ excitation current waveform structure.

A similar situation with an appearance of such asymmetric hysteresis loop is present in the coil of the permanent magnet motor, where permanent rotor magnet 
plays the role of the DC source. The results of a direct influence of the external magnetic field to the target coil have been reported in [8].

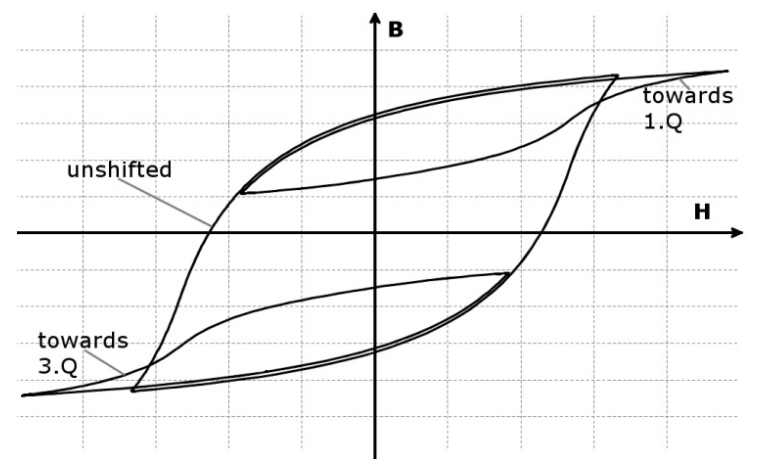

Fig. 1. Influence of an external magnetic field DC component on the hysteresis B-H loop shape.

Experimentally, such effect could be generated in several ways. This paper proposes a method that provides precise control of DC bias offset through the injection of DC current on the secondary side.

\section{DESCRIPTION OF EXPERIMENTAL MEASUREMENT SETUP}

For an emulation of the effect that represents a real excitation asymmetry of the transformer primary side, the experimental toroidal transformer is designed with a large number of primary turns (1072), along with a low number of secondary turns (20).

With such transformer, when a DC current is forced through the secondary coil, an additional DC magnetic field is injected in the transformer core. This approach eliminates the need for a direct superposition of large AC and low DC voltage sources, it preserves isolation between primary and secondary circuits and finally, DC current that is injected through the secondary side has to be in $\mathrm{mA}$ range in contrast with primary range of microamperes, which is more critical for the maintenance.

If the $\mathrm{B}-\mathrm{H}$ loop is shifted as described above, regular $\mathrm{AC}$ voltage excitation will produce an $\mathrm{AC}$ excitation current with a corresponding DC offset. For the system level analysis it is much easier to keep regular $\mathrm{AC}$ voltage excitation on the primary side with the DC current excitation placed on the secondary side. Such separation provides simple, decoupled injection of the DC component in the AC excitation current, which could be easily prepared and verified in the laboratory. The waveform of the primary AC excitation current remains the same, no matter which approach is applied.

TABLE I. TRANSFORMER PARAMETERS.

\begin{tabular}{|c|c|c|}
\hline & Primary coil & $\begin{array}{c}\text { Secondary } \\
\text { coil }\end{array}$ \\
\hline Number of turns & 1072 & 20 \\
\hline Nominal voltage true RMS [V] & 100.00 & 1.86 \\
\hline Core cross-section $\left[\mathrm{mm}^{2}\right]$ & \multicolumn{2}{|c|}{280} \\
\hline Magnetic path length [mm] & \multicolumn{2}{|c|}{144} \\
\hline Core material & \multicolumn{2}{|c|}{ M 4} \\
\hline Magnetizing current true RMS [mA] & \multicolumn{2}{|c|}{4.48} \\
\hline Nominal apparent power [VA] & \multicolumn{2}{|c|}{30} \\
\hline
\end{tabular}

The described setup is used as a controlled source of additional DC magnetic field in the transformer core. The related data of the experimental toroidal transformer are listed in Table I.

The experimental circuit schematic is illustrated in Fig. 2.

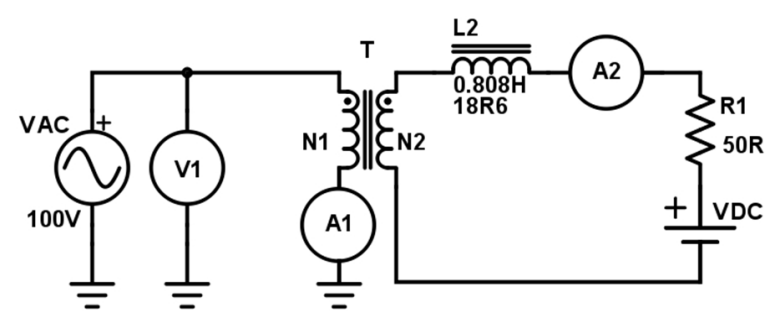

Fig. 2. Experimental schematic.

Relevant details about schematic components are listed in Table II.

TABLE II. SCHEMATIC DETAILS.

\begin{tabular}{|c|c|c|}
\hline Component & Nominal value & Description \\
\hline VAC & $100 \mathrm{~V}$ AC & Primary AC source \\
\hline V1 & Voltmeter & Oscilloscope voltage probe \\
\hline A1 & mA meter & Oscilloscope current probe \\
\hline T & Transformer & TABLE I \\
\hline L2 & $0.808 \mathrm{H}, 18 \mathrm{R} 6$ & Decoupling choke \\
\hline A2 & mA meter & Stand alone Ampermeter \\
\hline R1 & $50 \mathrm{R}$ & Additional resistance \\
\hline VDC & $0 \mathrm{~V}$ to $20 \mathrm{~V}$ & Adjustable DC source \\
\hline
\end{tabular}

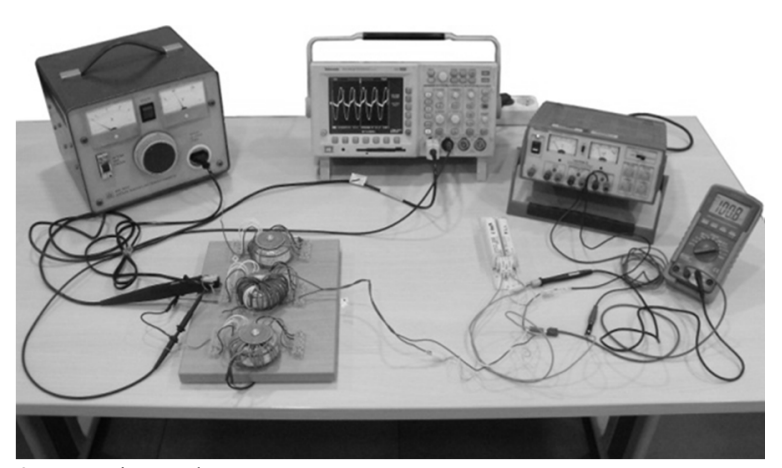

Fig. 3. Experimental setup.

As a measuring unit, the Tektronix TDS3054B (5 Gs/s, 4 channel) digital oscilloscope was used with the Tektronix TCP202 current probe, along with a hand-held universal instrument on $200 \mathrm{~mA}$ DC current range. The complete experimental setup bench is represented in Fig. 3.

\section{EXPERIMENTAL RESUlTS ANALYSIS}

For a succesful decomposition of an AC excitation current waveform to its unhysteretic (pure magnetization) and hysteretic components, the sampling process was performed with respect to zero crossing of the primary supply voltage at the instants when the voltage passes from negative to positive values, as it is represented in Fig. 4. Strictly, zero crossing should be synchronized to the secondary induced voltage, but, the voltage drop produced by $\mathrm{AC}$ excitation current in primary winding is negligible and therefore the assumption that both primary supplied and secondary induced voltages pass zero at the same instants with phase shift of $\pi$ can be accepted as correct.

The measurements were performed for 10 equidistant levels of DC bias to both negative and positive directions, 
and one measurement was performed without DC bias. All the measurements were done with the rated value of the primary AC supply voltage of $100 \mathrm{~V}, 50 \mathrm{~Hz}$ at a sampling rate of $100 \mathrm{KHz}$. In Fig. 5 the five of 21 experimentally obtained AC excitation current waveforms with DC bias of $100 \mathrm{~mA},-50 \mathrm{~mA}, 0 \mathrm{~mA}, 50 \mathrm{~mA}$ and $100 \mathrm{~mA}$ are represented. During transition from negative to positive DC bias values, at the $\mathrm{DC}$ bias value of $0 \mathrm{~mA}$, no demagnetization process of the transformer was performed, giving as a consequence smaller positive peak values toward the negative ones. This approach emulates a real situation in an $\mathrm{AC}$ grid, where a potential change of DC bias direction is not followed by any demagnetization process of the observed transformer at all.

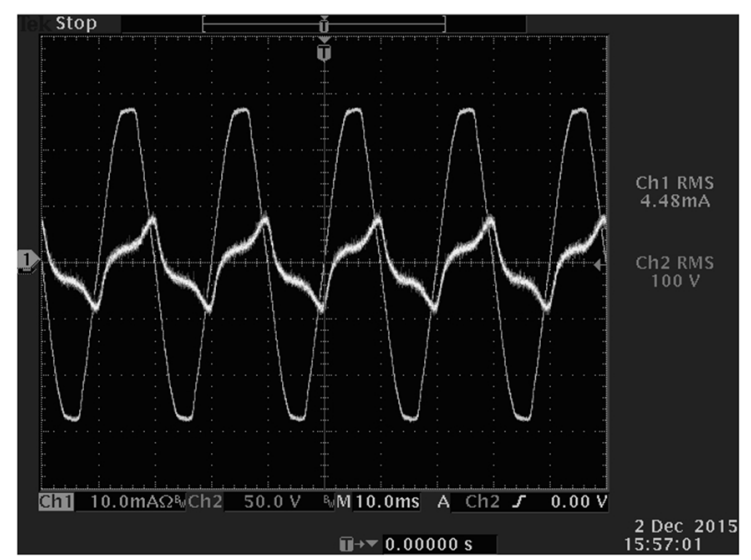

Fig. 4. Oscilloscope screenshot with the marked instant when voltage rise crosses zero.

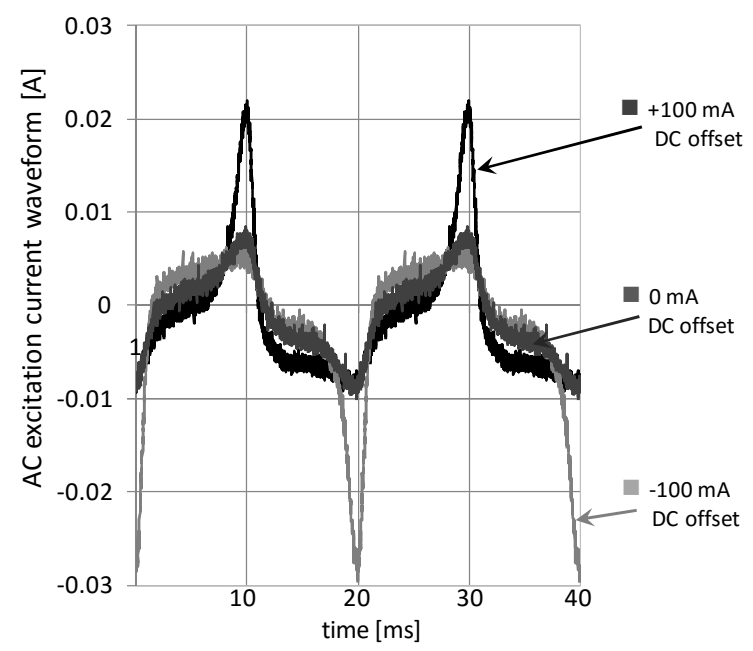

Fig. 5. AC excitation current waveforms with and without DC offsets.

The analysis was performed by using DFT over the sample sets of 2000 raw (non-filtered) samples. The amplitudes of harmonics of a higher order (above 20) were at the noise level, and therefore were rejected as less significant.

A very good linearity of harmonic amplitudes toward DC offset bias exists for the second order harmonic, as it is represented in Fig. 6. This linearity firmly correlates with the constant phase values, as it is represented in Fig. 7.

The same holds true for the 4-th order harmonic (Fig. 8). But, for the 6-th order harmonic, there is certain disturbance of the constant phase value (Fig. 9), and for the 8-th order harmonic this disturbance becomes pretty strong (Fig. 10).

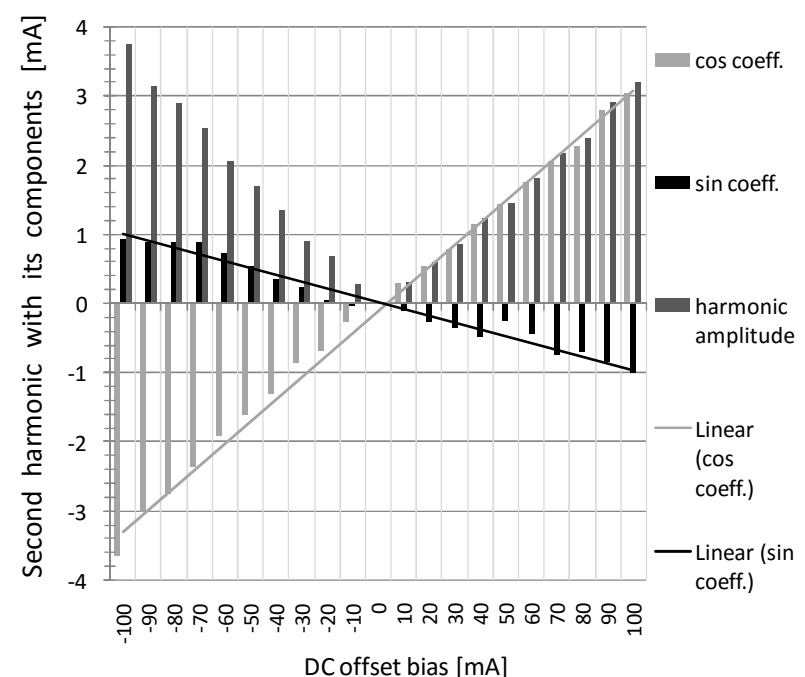

Fig. 6. Behaviour of the second order AC excitation current waveform harmonic and its cosine and sine components with respect to DC bias offsets.

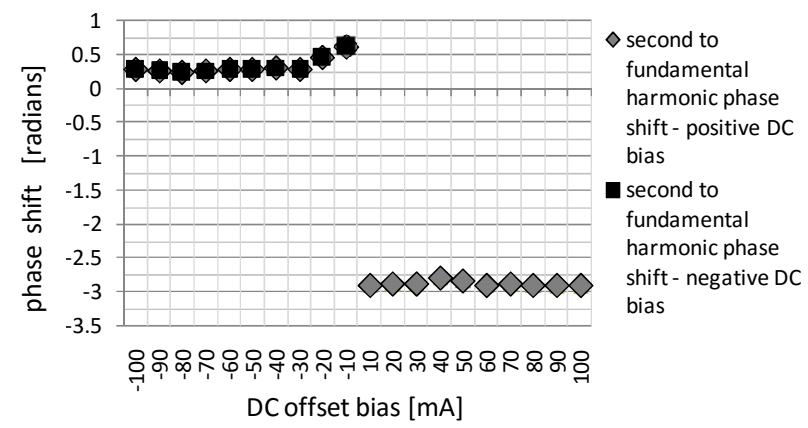

Fig. 7. Behaviour of the phase shift of the second order AC excitation current waveform harmonic with respect to DC bias offsets.

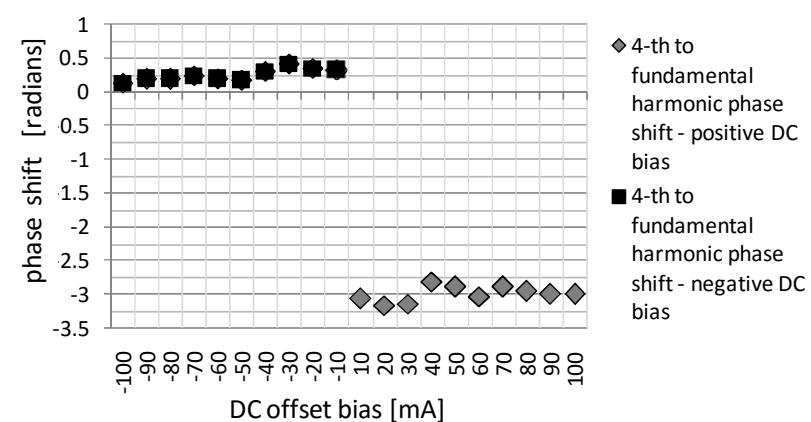

Fig. 8. Behaviour of the phase shift of the 4-th order AC excitation current waveform harmonic with respect to DC bias offsets.

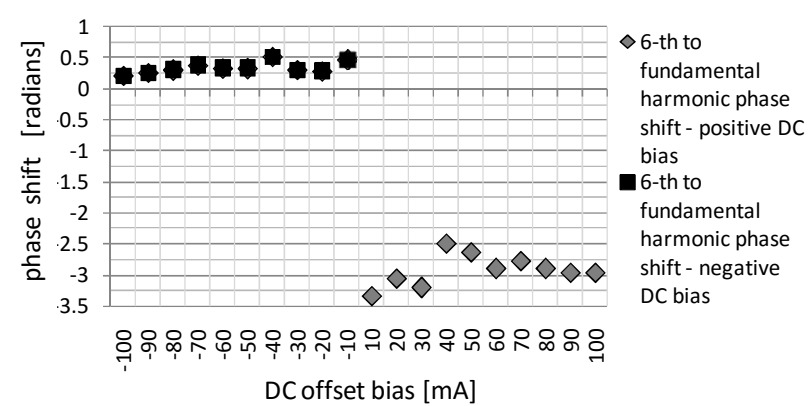

Fig. 9. Behaviour of the phase shift of the 6-th order AC excitation current waveform harmonic with respect to DC bias offsets.

For getting a straightforward insight concerning the behavior of the even AC excitation current waveforms harmonics, it is useful to include in the process of DFT analysis the next suitable decomposition of the AC 
excitation current waveform $i_{A C e x c}$ to its unhysteretic (pure magnetization) $i_{\text {unhyst }}$ and hysteretic $i_{\text {hyst }}$ components, as it is represented in Fig. 11.

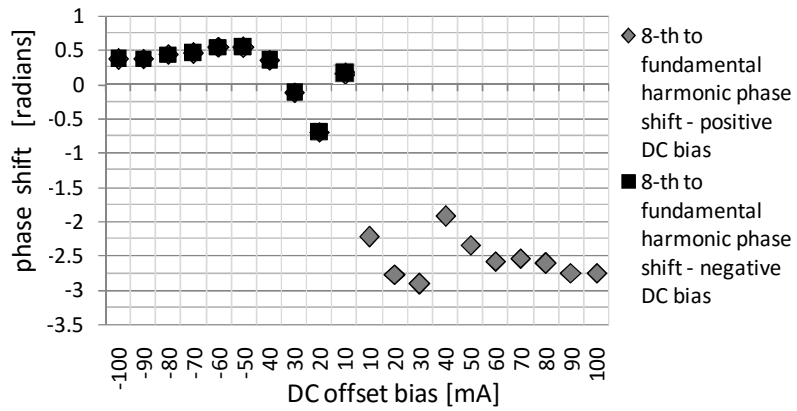

Fig. 10. Behaviour of the phase shift of the 8-th order AC excitation current waveform harmonic with respect to DC bias offsets.

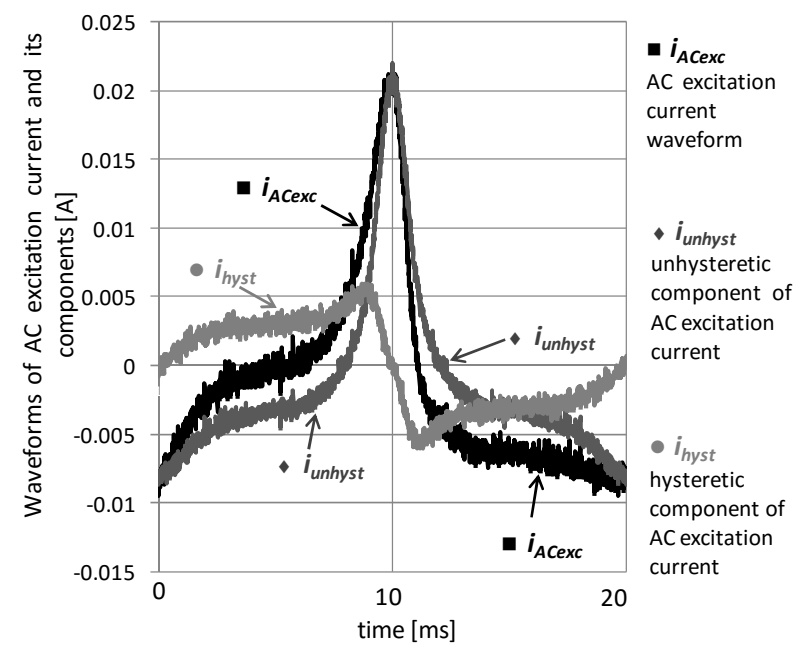

Fig. 11. Decomposition of AC excitation current $i_{A C e x c}$ waveform to its unhysteretic (pure magnetizing) $i_{\text {unhyst }}$ and hysteretic $i_{\text {hyst }}$ components.

This decomposition is performed by using the next definition expressions:

$$
i_{\text {unhyst }}(t)=0.5 \times\left(i_{A C e x c}(t)+i_{A C e x c}(T-t)\right),
$$

where $t \in[0, T]$, and

$$
i_{\text {hyst }}(t)=0.5 \times\left(i_{A C e x c}(t)-i_{A C e x c}(T-t)\right)
$$

where $t \in[0, T / 2]$.

$$
i_{\text {hyst }}(t)=-0.5 \times\left(i_{\text {ACexc }}(t)-i_{A C e x c}(T-t)\right),
$$

where $t \in[T / 2, T]$ that gives

$$
i_{\text {ACexc }}(t)=i_{\text {unhyst }}(t)+i_{\text {hyst }}(t) \text {, }
$$

where $t \in[0, T]$ with $\mathrm{t}=0$ denoting the zero crossing instant, whereas $\mathrm{t}=\mathrm{T}$ denotes the one $\mathrm{AC}$ excitation current period $\mathrm{T}=20 \mathrm{~ms}$ after the zero crossing instant $\mathrm{t}=0$. This decomposition of $\mathrm{AC}$ excitation current waveform is effectively used for the analysis of symmetric hysteresis loop curves, as it has been reported in [12].

The hysteretic AC excitation current component defined above has the phase shift zero relative to the primary supply voltage and reaches zero values at the primary supply voltage zero crossing instants

$$
i_{\text {hyst }}(0)=i_{\text {hyst }}(T / 2)=i_{\text {hyst }}(T)=0 \text {. }
$$

Therefore, the hysteretic AC excitation current component does not contribute to AC excitation current peak values at all, and the next relation holds true:

$$
\begin{gathered}
i_{A C \operatorname{exc}}(0)=i_{\text {unhyst }}(0)=i_{\text {unhyst }}(T)=i_{A C \operatorname{exc}}(T), \\
i_{A C \operatorname{exc}}(T / 2)=i_{\text {unhyst }}(T / 2),
\end{gathered}
$$

as it is represented in Fig. 11.

Furthermore, on the basis of the definition expressions (2),(3), DFT of the hysteretic AC excitation current component gives as a result the sine coefficients only and these are identical to the corresponding sine coefficients of the $\mathrm{AC}$ excitation current waveform, denoted as $\mathrm{B}_{\mathrm{k}}$. Similarly, on the basis of the definition expression (1), DFT of the unhysteretic AC excitation current component gives as a result the cosine coefficients only that are identical to the corresponding cosine coefficients of the AC excitation current waveform, denoted as $\mathrm{A}_{\mathrm{k}}$.

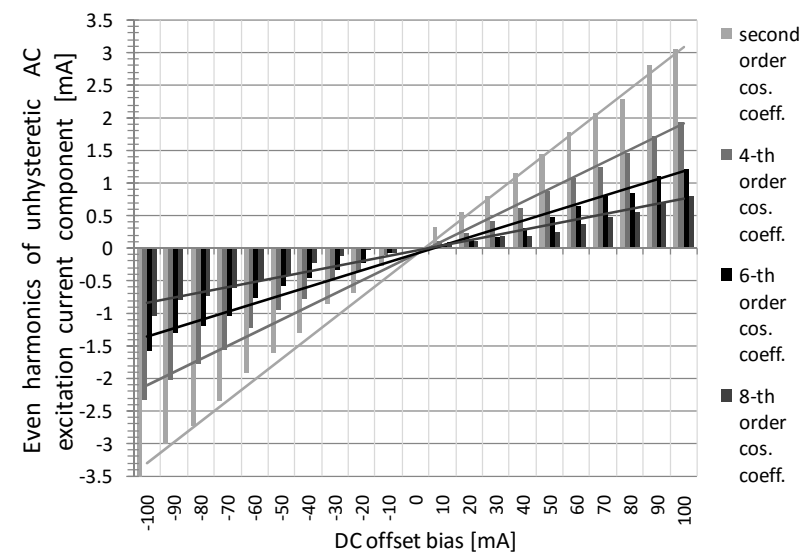

Fig. 12. Behaviour of the second, 4-th, 6-th and 8-th order harmonics of the unhysteretic (pure magnetizing) $\mathrm{AC}$ excitation current component with respect to DC bias offsets.

Therefore, the behavior of the even harmonics $\left(\mathrm{A}_{2 \mathrm{k}}\right)$ of the unhysteretic AC excitation current component only (pure magnetization current) can be observed, as it is represented in Fig. 12.

Evidently, for the unhysteretic (pure magnetizing) AC excitation current component, the firm linearity of its even harmonics remains preserved in spite of the high level of the noise presence.

Finally, DFT of separated hysteretic AC excitation current component exhibits interesting results concerning its even harmonics $\left(\mathrm{B}_{2 \mathrm{k}}\right)$ behaviour, as it is represented in Fig. 13.

Figure 13 presents, along with the even order harmonics, the corresponding trend-lines as the graphs of their 6-th degree approximation polynomials obtained by the least squares criterion. Undoubtedly, these harmonics do not exhibit a linear dependence relative to DC bias offset and this absence of linear dependence is not a consequence of the high presence of the noise. This nonlinearity is less present at the second and 4-th harmonics, but for 6-th, 8-th, 
and higher, it could not be ignored.

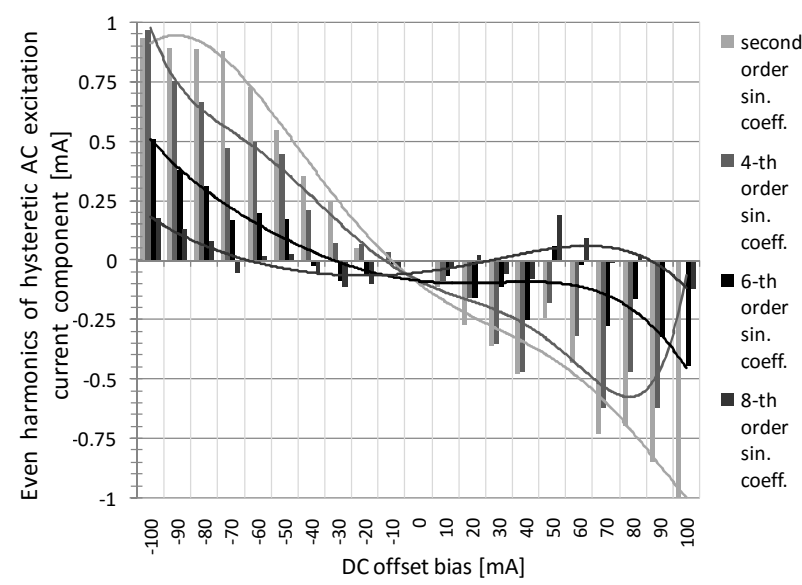

Fig. 13. Behaviour of the second, 4-th, 6-th and 8-th order harmonics of the hysteretic $\mathrm{AC}$ excitation current component with respect to DC bias offsets.

There is one more important result of the above proposed AC excitation current decomposition. Based on (6), (7) and since DFT of the unhysteretic AC excitation current component gives the cosine coefficients $\left(A_{k}\right)$ only, the following expressions are true for the $\mathrm{AC}$ excitation current peak values:

$$
\begin{gathered}
i_{A \operatorname{Cexc}}(0)=i_{A \operatorname{Cexc}}(T)=\sum_{k=0}^{n} A_{2 k}+\sum_{k=1}^{n} A_{2 k-1}, \\
i_{A \operatorname{Cexc}}(T / 2)=\sum_{k=0}^{n} A_{2 k}-\sum_{k=1}^{n} A_{2 k-1},
\end{gathered}
$$

with $\mathrm{n}$ denoting a half of an overall number of the considered harmonics. Also, for obtaining the peak to peak value, based on (6)-(9) the next expression holds true

$$
\begin{gathered}
i_{A C e x c}(T / 2)-i_{A C e x c}(0)= \\
=i_{A C \operatorname{exc}}(T / 2)-i_{A C e x c}(T)=-2 \sum_{k=1}^{n} A_{2 k-1},
\end{gathered}
$$

whereas for obtaining the peak difference value (the difference of positive peak value and the absolute value of negative peak value) states the next expression:

$$
\begin{gathered}
i_{A \operatorname{exc}}(T / 2)-\left|i_{A \operatorname{Cexc}}(0)\right|= \\
=i_{A C e x c}(T / 2)-\left|i_{A C e x c}(T)\right|, \\
i_{A \operatorname{exc}}(T / 2)+i_{A C \operatorname{exc}}(0)=2 \times \sum_{k=0}^{n} A_{2 k} .
\end{gathered}
$$

Table III shows the peak difference values and the peak to peak values of $\mathrm{AC}$ excitation current obtained by using expressions (11), (12) and (10) for all DC biases. In addition, Table III contains the measured true RMS AC excitation current values as a validity check toward the true RMS AC excitation current values obtained from the harmonics on the basis of the next expression

$$
I_{A C \operatorname{exc}}=\sqrt{A_{0}^{2}+0.5 \times \sum_{k=1}^{n} A_{k}^{2}+0.5 \times \sum_{k=1}^{n} B_{k}^{2}}
$$

All computations based on (10)-(13) were performed for the first 20 harmonics, along with the harmonic of zero order.

For the purpose of noise level evaluation, additional measurement is performed on the same setup without magnetizing current, i.e. primary $\mathrm{AC}$ voltage source switched off and bias DC current circuit disconnected. Such experiment produced measured current noise true RMS value of $767 \mu \mathrm{A}$, which if compared with AC current levels related to maximal and minimal DC offsets gives SNR in range from $15 \mathrm{~dB}$ to $20 \mathrm{~dB}$ respectively.

TABLE III. INJECTED SECONDARY DC BIAS CURRENT AND RELATED AC EXCITATION CURRENT DATA.

\begin{tabular}{|c|c|c|c|c|}
\hline $\begin{array}{c}\text { DC } \\
\text { offset } \\
\text { [mA] }\end{array}$ & $\begin{array}{c}\text { AC excitation } \\
\text { current true RMS } \\
\text { from ammeter } \\
\text { [mA] }\end{array}$ & $\begin{array}{c}\text { AC excitation } \\
\text { current true RMS } \\
\text { from harmonics } \\
\text { [mA] }\end{array}$ & $\begin{array}{c}\text { Peak } \\
\text { difference } \\
\text { from (8) } \\
\text { [mA] }\end{array}$ & $\begin{array}{c}\text { Peak to } \\
\text { peak from } \\
\text { (7) [mA] }\end{array}$ \\
\hline-100 & 7.96 & 7.90 & -23.27 & 32.76 \\
\hline-90 & 7.22 & 7.17 & -19.38 & 29.19 \\
\hline-80 & 6.86 & 6.83 & -18.07 & 27.34 \\
\hline-70 & 6.48 & 6.44 & -15.79 & 25.64 \\
\hline-60 & 5.95 & 5.94 & -13.08 & 22.53 \\
\hline-50 & 5.68 & 5.69 & -11.40 & 21.20 \\
\hline-40 & 5.31 & 5.25 & -9.30 & 18.86 \\
\hline-30 & 5.07 & 5.02 & -7.20 & 17.65 \\
\hline-20 & 4.93 & 4.93 & -6.52 & 16.78 \\
\hline-10 & 4.71 & 4.67 & -4.42 & 15.77 \\
\hline 0 & 4.48 & 4.45 & -1.66 & 15.53 \\
\hline 10 & 4.57 & 4.52 & -1.06 & 14.98 \\
\hline 20 & 4.65 & 4.63 & 1.24 & 15.28 \\
\hline 30 & 4.84 & 4.85 & 2.10 & 16.17 \\
\hline 40 & 5.06 & 5.06 & 2.50 & 17.45 \\
\hline 50 & 5.25 & 5.23 & 3.34 & 19.28 \\
\hline 60 & 5.66 & 5.67 & 5.08 & 21.71 \\
\hline 70 & 5.92 & 5.93 & 7.14 & 22.61 \\
\hline 80 & 6.22 & 6.14 & 8.31 & 24.10 \\
\hline 90 & 6.65 & 7.08 & 11.16 & 26.55 \\
\hline 100 & 7.05 & & & 28.88 \\
\hline
\end{tabular}

\section{CONCLUSIONS}

The experimental setup for a simple and precise control (injection and the measurement) of the primary current DC component has been presented and analysed in detail.

The main contribution of the presented work is the decomposition and interpretation of the $\mathrm{AC}$ excitation current through its hysteretic and unhysteretic components. The natural result of such decomposition is that only even harmonics of unhysteretic components provide all the information regarding the DC bias of the transformer. The DC bias level is directly mapped to the amplitudes of the second, fourth and following even harmonics, while the polarity of the DC bias is directly mapped to the even harmonic phases. It has been proved that it is sufficient to track only the second harmonic as a key pointer to the existence, level and direction of the DC offset, i.e. the excitation asymmetry.

As a follow-up to this research, the authors plan to work on the generalization of the asymmetry in the coil which should include general inductors and the coils of the permanent magnet electric machines (motors and generators). 


\section{REFERENCES}

[1] N. Petrovic, V. Pjevalica, V. Vujicic, "The theorem about the transformer excitation current waveform mapping into the dynamic hysteresis loop branch for the sinusoidal magnetic flux case", Serbian Journal of Electrical Engineering, vol. 12, no. 1, pp. 33-52, 2015. Online. [Available]: http://dx.doi.org/10.2298/SJEE1501033P

[2] L. Gertmar, P. Karlsson, O. Samuelsson, "On de injection to ac grids from distributed generation", in Proc. 11th European Conf. Power Electronics and Applications (EPE 2005), Dresden, Germany, 2005, pp. P1-P10. Online. [Available]: http://dx.doi.org/10.1109/EPE. 2005.219420

[3] S. Araujo, P. Zacharias, R. Mallwitz, "Highly efficient single-phase transformerless inverters for grid-connected photovoltaic systems", IEEE Trans. Ind. Electron., vol. 57, no. 9, pp. 3118-3128, 2010. Online. [Available]: http://dx.doi.org/10.1109/TIE.2009.2037654

[4] B. Zhang, X. Cui, R. Zeng, J. He, "Calculation of DC current distribution in AC power system near HVDC system by using moment method coupled to circuit equations", IEEE Trans. Magn., vol. 42, no. 4, pp. 703-706, 2006. Online. [Available]: http://dx.doi.org/10.1109/TMAG.2006.871460

[5] P. R. Price, "Geomagnetically induced current effects on transformers", IEEE Trans. Magn., vol. 17, no. 4, pp. 1002-1008, 2002. Online. [Available]: http://dx.doi.org/10.1109/TPWRD.2002.803710

[6] A. Rezaei-Zare, "Behavior of single-phase transformers under geomagnetically induced current conditions", IEEE Trans. Power Del. vol. 29, no. 2, pp. 916-925, 2014. Online. [Available]: http://dx.doi.org/10.1109/TPWRD.2013.2281516
[7] S. Hodder, B. Kasztenny, N. Fischer, Y. Xia, "Low second-harmonic content in transformer inrush currents - analysis and practical solutions for protection security", in Proc. Southern African Power System Protection Conf., Johannesburg, South Africa, 2014. [Online] Available: http://dx.doi.org/10.1109/CPRE.2014.6799037

[8] N. Pjevalica, M. Nikolic, I. Kastelan, "Analog circuitry for BLDC motor magnetic saturation diagnostic", in IEEE 18th Int. Symposium on Design and Diagnostic of Electronic Circuits and Systems, Belgrade, Serbia, 2015, pp. 287-290. Online. [Available]: http://dx.doi.org/10.1109/DDECS.2015.13

[9] N. U. Pjevalica, N. S. Petrovic, M. M. Nikolic, "Detection of the external magnetic field polarity through the inductance voltage harmonic phase order", in IEEE 23rd Telecommunications forum (TELFOR 2015), Belgrade, Serbia, 2015. Online. [Available]: http://dx.doi.org/10.1109/TELFOR.2015.7377539

[10] S. N. Vukosavic, "Detection and supression of parasitic DC voltages in 400V AC grids", FACTA UNIVERSITATIS, Series: Electronics and Energetics, vol. 28, no. 4, pp. 527-540, 2015. Online. [Available] http://dx.doi.org/10.2298/FUEE1504527S

S. N. Vukosavic, L. S. Peric, "High precision sensing of DC bias in AC grids", IEEE Trans. Power Delivery, vol. 30, no. 3, pp. 1179 1186, 2015, Online. [Available]: http://dx.doi.org/10.1109/ TPWRD.2014.2386257

[11] N. Petrovic, V. Pjevalica, N. Pjevalica, N. Teslic, "Analysis of symmetric hysteresis loop curves using the chebyshev nodes of the first kind", in 18th Int. Symposium on Power Electronics (Ee 2015), Novi Sad, Serbia, Paper No. DS1-T4-1, 2015, pp. 1-5. Online. [Available]: http://www.dee.uns.ac.rs/biblio/biblio-e.html 